Canadian

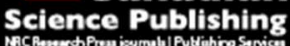

Applied Physiology, Nutrition, and Metabolism Physiologie appliquée, nutrition et métabolisme

\title{
Multi-modal High-Intensity Interval Training Increases Muscle Function and Metabolic Performance in Females
}

\begin{tabular}{|r|l|}
\hline Journal: & Applied Physiology, Nutrition, and Metabolism \\
\hline Manuscript ID & apnm-2015-0238.R1 \\
\hline Manuscript Type: & Article \\
\hline Complete List of Authors: & $\begin{array}{l}\text { Buckley, Stephanie; University of Saskatchewan, Physical Therapy } \\
\text { Knapp, Kelly; University of Saskatchewan, Physical Therapy } \\
\text { Lackie, Amy; University of Saskatchewan, Physical Therapy } \\
\text { Lewry, Colin; University of Saskatchewan, Physical Therapy } \\
\text { Horvey, Karla; University of Saskatchewan, Physical Therapy } \\
\text { Benko, Chad; Synergy Strength and Conditioning, ; BOSS Strength } \\
\text { Institute, } \\
\text { Trinh, Jason; Synergy Strength and Conditioning, } \\
\text { Butcher, Scotty; University of Saskatchewan, Physical Therapy; BoSS } \\
\text { Strength Institute, }\end{array}$ \\
\hline Keyword: & $\begin{array}{l}\text { sprint interval training, intermittent training, strength training < exercise, } \\
\text { aerobic power, HIIT }\end{array}$ \\
\hline &
\end{tabular}




\section{Multi-modal High-Intensity Interval Training Increases Muscle Function and Metabolic Performance in Females}

Stephanie Buckley, Kelly Knapp, Amy Lackie, Colin Lewry, Karla Horvey, Chad Benko, Jason Trinh, Scotty Butcher

\section{Corresponding Author:}

Dr. Scotty Butcher, PhD, PT, ACSM-RCEP

1121 College Dr., Saskatoon, SK, Canada, S7N 0W3

Email: scotty.butcher@usask.ca

Phone: 306-966-1711

Fax: 306-966-6575

\section{Author Affiliations and addresses:}

Stephanie Buckley: School of Physical Therapy, University of Saskatchewan; slb589@mail.usask.ca

Kelly Knapp: School of Physical Therapy, University of Saskatchewan; krk201@mail.usask.ca

Amy Lackie: School of Physical Therapy, University of Saskatchewan; amy.lackie@usask.ca

Colin Lewry: School of Physical Therapy, University of Saskatchewan; cg1515@mail.usask.ca

Karla Horvey: School of Physical Therapy, University of Saskatchewan; karla.horvey@usask.ca

Chad Benko: Synergy Strength and Conditioning, Saskatoon, SK; BOSS Strength Institute, Saskatoon, SK; chad@synergystrength.ca

Jason Trinh: Synergy Strength and Conditioning, Saskatoon, SK; jhktron@gmail.com

Scotty Butcher: School of Physical Therapy, University of Saskatchewan; BOSS Strength Institute, Saskatoon, SK; scotty.butcher@usask.ca 


\section{Abstract:}

High intensity interval training (HIIT) is a time efficient method of improving aerobic and anaerobic power and capacity. In most individuals, however, HIIT using modalities such as cycling, running, and rowing does not typically result in increased muscle strength, power, or endurance. The purpose of this study is to compare the physiological outcomes of traditional HIIT using rowing (Row-HIIT) with a novel multi-modal HIIT (MM-HIIT) circuit incorporating multiple modalities, including strength exercises, within an interval. Twenty-eight recreationally active, females (age 24.7 \pm 5 .4years) completed six-weeks of either Row-HIIT or MM-HIIT and were tested on multiple fitness parameters. MM-HIIT showed similar improvements $(\mathrm{p}<0.05$ for post hoc pre-post training increases for each group) as Row-HIIT in $\mathrm{VO}_{2} \mathrm{max}$ (7 vs 5\%), anaerobic threshold (13 vs 12\%), respiratory compensation threshold (7 vs 5\%), anaerobic power (15 vs $12 \%$ ), and anaerobic capacity (18 vs 14\%, respectively). The MM-HIIT group had significant ( $\mathrm{p}<0.01$ for all) increases in squat $(39 \%)$, press $(27 \%)$, and deadlift $(18 \%)$ strength, broad jump distance (6\%), and squat endurance (280\%); whereas the Row-HIIT group had no increases in any muscle performance variable ( $\mathrm{p}$ values from $0.33-0.90$ ). Post testing, MM-HIIT was greater than Row-HIIT for 1RM squat $(64.2 \pm 13.6$ vs. $45.8 \pm 16.2 \mathrm{~kg}, \mathrm{p}=0.02), 1 \mathrm{RM}$ press $(33.2 \pm 3.8$ vs. $26.0 \pm 9.6 \mathrm{~kg}, \mathrm{p}=0.01)$, and squat endurance $(23.9 \pm 12.3$ vs. $10.2 \pm 5.6 \mathrm{reps}, \mathrm{p}<0.01$ respectively). MM-HIIT training resulted in similar aerobic and anaerobic adaptations, but greater muscle performance increases than Row-HIIT in recreationally active females.

Key words: sprint interval training, intermittent training, strength training, aerobic power, HIIT, multi-modal, rowing 


(n)

\section{Introduction}

High intensity interval training (HIIT) consists of repeated execution of high-intensity exercise interspersed with recovery periods of low intensity exercise or complete rest (Laursen et al. 2010). HIIT has been shown to increase aerobic and anaerobic performance within a low time commitment (Bayati et al. 2011; Hazell et al. 2010). HIIT results in physiological benefits including improvements in aerobic capacity, cardiorespiratory fitness, glucose tolerance, exercise endurance, skeletal muscle oxidative capacity, glycogen content, and reductions in the rate of lactate production and glycogen utilization (Gibala et al. 2010; Nybo et al. 2010; Weston et al. 2014). Nybo et al. (2010) reported that HIIT resulted in a significant reduction in arterial blood pressure and superior improvements in cardiorespiratory fitness, as indicated by increases in $\mathrm{VO}_{2} \mathrm{max}$, compared to traditional prolonged training.

In contrast to the evidence supporting metabolic adaptations with HIIT, there is less evidence evaluating the muscle strength benefits of HIIT. It is well known that strength training induces muscle hypertrophy and strength increases (Nybo et al. 2010). Skeletal muscle strength has been shown to be an important determinant of functional capacity (Verdijk et al. 2009). Typically, HIIT does not have a significant long term impact on muscle mass or parameters indicative of skeletal health (Astorino et al., 2012; Nybo et al. 2010). Thus, the addition of a strength training program to a HIIT program would be required to additionally optimize muscle function, but would require excessive time to complete both aspects of training (Astorino et al. 2012). 
One potential solution that has emerged to reduce the time required for multiple

2 adaptations is multi-modal (MM) training. MM training uses a variety of resistance, body-

3 weight, and/or traditional conditioning modalities (eg. sprinting, rowing, kettlebell swings,

4 battle-ropes) performed either in a continuous circuit (Myers et al. 2015) or interval (McRae et

5 al. 2012) format. While the continuous circuit methodology utilizes multiple modalities within a

6 single session, previous work with interval style MM training has limited individual sessions to a

7 single body-weight modality (McRae et al. 2012) and rotated modalities between sessions. While

8 this format is effective for inducing aerobic, aerobic, and muscle endurance adaptations (McRae

9 et al. 2012), the lack of a whole body strength component within each interval may limit the

10 muscle performance adaptations.

11 In this study, we examine a novel multi-modal HIIT (MM-HIIT) protocol that

12 incorporates resistance and conditioning modalities within the work interval to combine multiple

13 training effects while minimizing training time. This form of training is common in many fitness

14 facilities, but has not undergone scientific investigation. The purpose of this study is to document

15 the physiological benefits of a MM-HIIT program compared with HIIT using a more traditional

16 aerobic modality (rowing). First, we hypothesize that both the traditional HIIT using rowing

17 (Row-HIT) and MM-HIIT groups will improve similarly in aerobic and anaerobic energy

18 systems. Our second hypothesis is that the MM-HIIT group will improve to a greater degree in

19 strength, muscle power, and muscle endurance compared to the Row-HIIT.

\section{Materials and methods}

22 Study Design 
A prospective longitudinal group randomized trial study design was implemented.

2 Participants were grouped using convenience assignment based on their availability for training

3 times and each group was then randomly assigned to complete either the Row-HIIT or the MM-

4 HIIT training program. On day one, all participants underwent screening and initial testing by a

5 physical therapist, which consisted of a medical history, baseline blood pressure, height and

6 weight, and a treadmill $\mathrm{VO}_{2}$ max test. Following day one of screening, participants engaged in

7 three familiarization, practice, and adaptation sessions for the movements to be used during

8 training and testing. Familiarization was followed by three field testing sessions conducted

9 during week \#2. Participants then underwent 6 weeks of group training 3 times a week. Posttesting was then performed on the same measures as the pre-training tests. For the full testing,

11 familiarization, and training program schedules, please see Supplementary Material (Tables S1

12 and S2). Individual participant attendance was recorded and data were accepted if participants achieved greater than $80 \%$ attendance. Test evaluators were blinded to the group randomization.

Participants

Thirty two recreationally active participants between the ages of 18 and 35 were recruited

17 and gave consent to the study, which was approved by the local institutional ethics review board. Recreationally active was defined as participants engaging in periodic physical activity or

19 exercise between 1 and 3 hours weekly for 1 month or longer. Participants were not currently engaging in systematic endurance or weight training. Study participants were instructed to stay

21 consistent with their levels of exercise activity prior to the study, but to not progress these

22 activities. Participants were excluded if they reported any acute or chronic cardiovascular, 
1 metabolic, respiratory, or other conditions that would have prevented them from participating in

2 heavy exercise. In addition, if adverse findings were recorded during screening that would have

3 been associated with an increased risk during exercise, participants were excluded from the

4 study. Sixteen participants were assigned to each training group at baseline. Three participants

5 (two from the Row-HIIT and one from the MM-HIIT group) withdrew consent prior to the

6 initiation of the training phase. One participant in the MM-HIIT group dropped out of the study

7 due to an injury obtained outside of the study. There were no other dropouts for any reason.

8 Thus, fourteen participants in each group completed the study (Table 1).

9

Tests and Measures

$\mathrm{VO}_{2} \max$ test

Maximal aerobic power $\left(\mathrm{VO}_{2} \max \right)$ was assessed using a customized graded treadmill protocol. Participants completed a three minute warm up at $3.5 \mathrm{kph}$ and $1 \%$ grade on a treadmill (TrackMaster TMX425C, Full Vision, Newton, KS), which was followed by incremental one minute stages. In the initial stages, speed was progressed by $1 \mathrm{kph}$ per minute with the grade held constant at $1 \%$ until the participant indicated they had reached a pace slightly greater than their subjectively determined sustainable running speed. At this point, the speed was held constant and grade was increased by $2 \%$ per minute until voluntary exhaustion. Heart rate (HR) was measured continuously with a chest strap (Polar Electro Canada, Lachine, QC). Ventilatory gases were measured and analysed with a commercial metabolic measurement system (TrueOne 2400, ParvoMedics, East Sandy, UT). Rate of perceived exertion (RPE) was recorded every two minutes and at peak exercise using a 10-point Borg scale (Noble et al. 1983). $\mathrm{VO}_{2} \mathrm{max}$ was 
1 considered to be obtained if two of the following criteria were obtained; (1) HR was $\geq 90 \%$ of 2 predicted maximum (220 - age), (2) $\mathrm{RER}\left(\mathrm{VCO}_{2} / \mathrm{VO}_{2}\right) \geq 1.10$, and (3) $\mathrm{RPE} \geq 9 / 10$. Data was 3 reported in 20 second averages. In addition to aerobic power, the anaerobic (AT) and respiratory compensation (RCT)

5 thresholds were determined by analysis of ventilatory gases. AT was defined as the breakpoint

6 in ventilation $\mathrm{V}_{\mathrm{E}} / \mathrm{VO}_{2}$ while $\mathrm{V}_{\mathrm{E}} / \mathrm{VCO}_{2}$ had plateaued and RER was between 0.98 and 1.02. RCT 7 was defined as the subsequent breakpoint in $\mathrm{V}_{\mathrm{E}} / \mathrm{VCO}_{2}$ (Simon et al. 1983).

9 Wingate Anaerobic Test Anaerobic power and capacity were determined by a 30 second Wingate Anaerobic Test

11 (WAT) as per standard protocol (Bar-Or, 1987). A Monark 894E cycle ergometer was used and 12 the resistance was set at $0.075 \mathrm{~kg} / \mathrm{kg}$ body mass. The test was preceded by a practice drop for 3 13 seconds at $0.03 \mathrm{~kg} / \mathrm{kg}$ body mass and a 1 minute rest period. Power was calculated using 14 standard Monark Anaerobic Testing software. Anaerobic power was determined to be the 15 highest 5 second mean power. Anaerobic capacity was determined to be the mean power over 16 the entire 30 seconds.

Muscle strength

On separate days, muscle strength was assessed using 1RM testing for back squat, press, and deadlift using standardized protocol (Baechle \& Earle, 2008). The back squat was

21 performed to below parallel (hip crease below the apex of the patella), the press was initiated 22 with the bar on the clavicles, and the deadlift was performed in the conventional stance. 
2 Muscle endurance and power

3 Muscle endurance was assessed by repetitive back squats to voluntary exhaustion. The

4 load was set at $70 \%$ of the pre-training $1 \mathrm{RM}$ back squat for both pre- and post-training tests.

5 Movement standards were the same as for the 1RM test and the total successful repetitions

6 achieved without a repetition rest were recorded. Muscle power was assessed using a static

7 broad jump. The best horizontal distance achieved out of three trials was recorded.

8

$9 \quad$ Training Program

10 During the 6 week training program, participants attended group training sessions for 60

11 minutes 3 times a week. The 60 minute training period was broken down into 20 minutes of

12 active warm up, 24 minutes of training and 16 minutes of cool down. It is important to note that

13 both the Row-HIIT and MM-HIIT groups were given the same instructions to perform all-out

14 intensity every interval (ie. achieve an RPE of 9 or 10/10 for each interval).

15

16 Rowing (Row-HIIT)

17 Participants randomized in the Row-HIIT group performed 60 seconds of all-out intensity

18 rowing intervals every 4 minutes with 3 minutes of rest (passive recovery) for a total of 6 rounds.

19 Participants were instructed to engage in all-out intensity during the work period in order to

20 achieve maximum distance on each interval.

21

22 MM-HIIT 
Participants randomized into the MM- HIIT group also performed 60 seconds of all-out

2 intensity work every 4 minutes with a 3 minute rest in between for 6 rounds. Each multi-modal

3 HIIT interval was broken down into 3 parts: a strength exercise for 4-6 repetitions, an accessory

4 movement for 8-10 repetitions, and a metabolic component conducted all out for the remainder

5 of the 60 seconds. For the strength exercise and accessory movement, participants were

6 instructed to load heavy, with the purpose of fatiguing by the end of the intervals. For example,

7 for the strength exercise, a "heavy" load was defined as 4-6 repetitions and if participants were

8 able to sustain 6 repetitions across all 6 intervals, the load would be progressed in the next

9 session using the same exercise. Participants were instructed to engage in all-out intensity

10 during every interval. Please see Supplementary Material (Table S2) for more information.

12 Statistical Analysis

Statistica V8.0 (StatSoft Inc, Tulsa OK) was used to analyze the data. Means and

14 standard deviations were reported for each descriptive variable. A two-way, repeated measures

15 factorial ANOVA was used to analyse the main effects for each of the main outcomes $\left(\mathrm{VO}_{2} \mathrm{max}\right.$

16 test, Wingate test, and muscle strength/power/endurance tests). Where significant main effects

17 were observed, Tukey's post hoc analysis was performed to determine specific differences.

18 Percent attendance was compared between groups using an unpaired t-test. Alpha was set $a$

19 priori to 0.05 .

20

$21 \quad$ Results

22 Attendance 
1 All participants who completed the study performed greater than $80 \%$ of the training sessions.

2 There was no difference in attendance between groups ( $89 \pm 5$ and $91 \pm 6 \%$, for MM-HIT and

3 Row-HIIT, respectively; $\mathrm{p}=0.69)$.

4

$5 \mathrm{VO}_{2} \max$ test

$6 \quad$ All participants achieved the criteria for attaining $\mathrm{VO}_{2}$ max and reached tolerance at 8:13

$7 \pm 0: 44$ minutes:seconds for the pre-training test and 10:36 $\pm 1: 21$ minutes:seconds for the post-

8 training test. There were no interaction effects for the $\mathrm{VO}_{2}$ values at maximum, AT, or RCT.

9 There was a significant main effect of time, but not for group, for each of $\mathrm{VO}_{2} \max (p<0.01)$,

$10 \mathrm{VO}_{2}$ at $\mathrm{AT}(p<0.01)$ and $\mathrm{VO}_{2}$ at $\mathrm{RCT}(p<0.01)$. Table 2 shows the individual group data and

11 indicates that there were increases in $\mathrm{VO}_{2} \mathrm{max}$ and $\mathrm{VO}_{2}$ at $\mathrm{AT}$ for both groups, but $\mathrm{VO}_{2}$ at RCT

12 increased only in the MM-HIIT group. There were no significant post-training differences

13 between groups for $\mathrm{VO}_{2} \max (p=0.99), \mathrm{VO}_{2}$ at $\mathrm{AT}(p=0.09)$, or $\mathrm{VO}_{2}$ at $\mathrm{RCT}(p=0.08)$.

14

15

16

17

1

22 Strength

Wingate test

There were no interaction effects for anaerobic power or capacity. There were significant main effects for time, but not group, for both anaerobic power $(p<0.01)$ and capacity $(p<$

0.01). As shown in Table 2, there were significant increases in anaerobic power and capacity for both groups with no post-training differences between the Row-HIIT and MM-HIIT groups for anaerobic power $(p=0.643)$ and capacity $(p=0.825)$. 
There were significant interaction effects for $1 \mathrm{RM}$ squat $(p<0.01)$ and $1 \mathrm{RM}$ press $(p<$

$20.01)$. There were also significant main effects for time in squat, press, and deadlift $(p<0.01)$,

3 and for group in squat and press $(p<0.01)$. Post hoc analysis revealed that the MM-HIT group

4 increased strength in the squat $(p<0.01)$, press $(p<0.01)$, and deadlift $(p<0.01)$, whereas the

5 Row-HIIT group did not (Figure $1 ; p=0.69,0.82$, and 0.33 , respectively). There were

6 significant post-training group differences for the $1 \mathrm{RM}$ squat $(p=0.02)$, and the $1 \mathrm{RM}$ press $(p$

$7=0.01)$, but not the 1 RM deadlift $(p=0.99)$.

$9 \quad$ Muscle power and endurance

There was a significant interaction for squat endurance $(p<0.01)$. There were significant

11 main effects for time for both the broad jump $(p<0.01)$ test and the squat endurance test $(p<$

12 0.01). Figure 2 shows that the MM-HIIT group increased distance achieved in the broad jump

13 (Figure $2 \mathrm{a} ; p<0.01$ ) and the number of reps completed on the squat endurance test (Figure $2 \mathrm{~b} ; p$

$14<0.01)$, whereas the Row-HIIT group did not ( $p=0.90$ and 0.88 , respectively). Post hoc testing revealed there was a significant difference in post-training values between groups for the squat

16 endurance test $(p<0.01)$, but not the broad jump $(p=0.99)$.

\section{Discussion}

We examined the effects of a novel MM-HIIT protocol compared with Row-HIIT on aerobic and anaerobic performance, and muscle strength, power, and endurance. During the 6

21 week training period, both MM-HIIT and Row-HIIT increased anaerobic threshold (13\% vs

$2212 \%), \mathrm{VO}_{2} \max (7 \%$ vs $5 \%)$, and anaerobic power (15\% vs $\left.12 \%\right)$ and capacity (18\% vs $14 \%$, 
respectively). Only the MM-HIIT group had significant increases in RCT (7\%). These findings support our first hypothesis that both Row-HIIT and MM-HIIT will have similar adaptations in aerobic and anaerobic energy systems.

In terms of muscle strength, power, and endurance, only MM-HIIT resulted in significant improvements in squat, deadlift, and overhead press strength. Similarly, significant increases in muscle power (broad jump) and endurance (70\% 1RM backsquat) were only observed in the MM-HIIT group. These findings support our second hypothesis that the MM-HIIT group would improve muscle performance to a greater degree than Row-HIIT.

Traditional HIIT has consistently shown increases in aerobic and anaerobic performance compared to continuous endurance exercise (Gist et al., 2013; Hazell et al., 2010; Tabata et al., 1996). Although our study did not directly compare HIIT with continuous exercise, our findings are similar in regard to aerobic and anaerobic performance adaptations for both methods of HIIT training. Increases due to HIIT of $4-13 \%$ for $\mathrm{VO}_{2} \mathrm{max}$ and $4-12 \%$ for anaerobic performance have been observed previously. (Cook et al., 2010; Driller et al., 2009; Gist et al., 2013; Hazell et al., 2010; Ronnestad et al., 2015; Sloth et al., 2013) These changes are similar to the degree of adaptation we observed with both protocols, indicating that our novel MM-HIIT and the rowing HIIT training are similarly effective for metabolic system changes. In contrast, traditional HIIT does not typically increase muscle strength (Astorino et al., 2012; Nybo et al., 2010) and this is supported by the present findings. We observed, however, increases in muscle strength, power, and endurance with our MM-HIIT protocol that are not typically observed with traditional HIIT. There are a limited number of studies reporting the effects of multi-modal training on multiple aspects of performance. McRae et al. (2012) studied the effects of HIIT using whole 
1 body movements (burpees, jumping jacks, mountain climbers, or squat thrusts) rotated across a

2 four week training period, but did not incorporate higher resistance activities or different

3 movements within each interval. They demonstrated similar improvements in aerobic capacity

4 with their HIIT protocol than with continuous treadmill endurance training, but exhibited greater

5 benefits to muscular endurance (40-200\% increases). Myers et al. (2015) studied the effects of

6 five weeks of training using a continuous MM circuit protocol, which rotated exercises and

7 modalities within and between sessions, compared with a combined strength and aerobic training

8 protocol. They demonstrated superior aerobic changes with their circuit protocol (11\% in

$\left.9 \mathrm{VO}_{2} \max \right)$ but similar changes in anaerobic power and capacity ( 5 and $3 \%$, respectively) compared with the combined program. In terms of muscle strength, while the combined group

11 increased all measures, the MM circuit group increased only vertical chest press (21\%) and

12 hamstring curl (8\%) strength, but not lat pull down and knee extension strength. In addition, the

13 circuit group was inferior in their measures of muscle endurance; although they compared the

14 number of repetitions completed at $60 \%$ of $1 \mathrm{RM}$ on each strength exercise at the corresponding

15 time point, rather than using the pre-training 1RM for both tests. In comparison, our study of

16 MM-HIIT resulted in increases in strength of 39\% for the 1 RM back squat, $27 \%$ for the 1 RM

17 overhead press, and $18 \%$ for the 1 RM deadlift, in power of $6 \%$ for the broad jump distance, and

18 also of muscle endurance of $280 \%$ for the squat endurance, demonstrating a large increase in

19 multiple measures of muscle performance with our protocol, in addition to the aerobic and

20 anaerobic adaptations. While direct comparison between our protocol and that of Myers and

21 colleagues (2015) and McRae and colleagues (2012) cannot be conclusive due to methodological

22 differences between studies, our protocol appears to be at least similar, or in some cases greater, 
1 for adaptations across multiple measures of fitness. Future work should examine the adaptations

2 achieved with differing MM protocols.

Combining HIIT with strength training is typically achieved by participants performing

4 two simultaneous training protocols (concurrent training) and requires increased time to

5 complete. Wong et al. (2010) demonstrated that professional soccer players concurrently

6 training in HIIT and muscular strength had significantly greater improvements in $10 \mathrm{~m}$ and $30 \mathrm{~m}$

7 sprint times, vertical jump height and maximal muscle strength (back half squat and bench press)

8 compared to training in soccer alone, but required increased training time. Concurrent training

9 involving sprint interval and strength training has been shown to result in similar upper and

10 lower body strength gains compared with strength training alone, with the addition of greater

11 increases in $\mathrm{VO}_{2}$ max. The findings of Wong et al. (2010), and those of others, (Cantrell et al.,

12 2014; deSouza et al., 2013; McNamara et al., 2013) suggest that concurrent training with HIIT

13 does not attenuate the strength response. In comparison to traditional concurrent training, our

14 study displayed improvements in aerobic and anaerobic performance, along with improvements

15 in muscle strength, power and endurance with MM-HIIT without the addition of excess training

16 time. Based on these findings, MM-HIIT is a time efficient training method in order to achieve

17 metabolic and muscular adaptations; however, further study is required to determine if a MM-

18 HIIT protocol can maintain the same degree of strength adaptations as more traditional

19 concurrent protocols or to strength training alone. In addition, as the generalizability of our study

20 is limited to young, healthy, active females without specific training experience, future study

21 should examine the effects of MM-HIIT in sedentary individuals, males, and older adults or

22 those with chronic conditions. 
2 in a limited time frame are of interest to rehabilitation, health, and exercise professionals.

3 Traditional methods of HIIT are time-efficient protocols resulting in multiple health and

4 aerobic/anaerobic performance benefits (Gibala et al. 2012), but usually lack adaptations in

5 muscle strength, power, and/or endurance. Our novel MM-HIIT protocol results in similar

6 aerobic and anaerobic performance adaptations to that of traditional HIIT protocols, but has the

7 additional benefit of improvements in muscle strength, power and endurance in recreationally

8 active females.

9

\section{Conflict of Interest Statement}

11 Mr. Benko is co-owner of, and Dr. Butcher an unpaid consultant with, Synergy Strength and

12 Conditioning, where the research was conducted.

\section{Acknowledgements}

15 The authors acknowledge the technical assistance of Evelyn Pata. 


\section{References}

(n)

Astorino, T.A., Allen, R.P., Roberson, D.W., and Jurancich, M. 2012. Effect of highintensity interval training on cardiovascular function, $\mathrm{VO}_{2} \mathrm{max}$, and muscular force. J. Str. Cond. Res. 2012: 26(1): 138-145.

Baechle, T.R., and Earle, R.W. 2008. Essentials of strength training and conditioning, $3^{\text {rd }}$ ed. NSCA National Strength \& Conditioning Association. Human Kinetics, Champaign, IL.

Bar-Or, O. 1987. The Wingate anaerobic test: An update on methodology, reliability and validity. Sports Med. 4: 381-394.

Bayati, M., Fazard, B., Gharakhanlou, R., and Agha-Alinejad, H. 2011. A practical model of low-volume high-intensity interval training induces performance and metabolic adaptations that resemble 'all-out' sprint interval training. J. Sports Sci. Med. 10: 571-576.

Cantrell G. S., Schilling B.K., Paquette M.R., and Murlastis Z. 2014. Maximal strength, power, and aerobic endurance adaptations to concurrent strength and sprint interval training. Eur. J. Appl. Physiol. 114: 763-771.

Cook K., Cathcart A.J., Scott R.A., and Easton C. 2010. High intensity interval training increases aerobic and anaerobic capacity in collegiate female soccer players. Med. Sci. Sport Exerc. 42: 698-699.

Driller, M.W., Fell J.W., Gregory J.R., Shing C.M., and Williams A.D. 2009. The effects of high-intensity interval training in well-trained rowers. Int. J. Sports Physiol. Perform. 4: 110121. 
de Souza, E.O., Tricoli, V., Roschel, H., Brum, P.C., Bacurau, A.V.N., Ferreira, J.C.B.,

Aoki, M.S., Neves-Jr, M., Aihara, A.Y., da Rocha Correa Fernandes, A., Ugrinowitsch, C. 2013.

Molecular adaptations to concurrent training. Int. J. Sports Physiol. Perform. 34: 207-213.

Gibala, M.J., Little, J.P., MacDonald, M.J., and Hawley, J.A. 2012. Physiological

adaptations to low-volume, high-intensity interval training in health and disease. J. Physiol. 590:

$6 \quad 1077-1084$

Gist N.H., Fedewa M.V., Dishman R.K., and Cureton K.J. 2013. Sprint interval training effects on aerobic capacity: A systematic review and meta-analysis. Sports Med. 44: 269-279. sprint interval training bouts enhance both aerobic and anaerobic performance. Eur. J. Appl.

11 Physiol. 110: 153-160. volume training? Scand. J. Med. Sci. Sports. 20: 1-10. periodization, and maximal-effort cycling on strength and power. J. Strength Cond. Res. 27: $1463-1470$. McRae G., Payne A., Zelt J.G.E., Scribbans T.D., Jung M.E., Little J.P., and Gurd B.J. 2012. Extremely low volume, whole-body aerobic-resistance training improves aerobic fitness 19 and muscular endurance in females. Appl. Physiol. Nutr. Metab. 37: 1124-1131. 
2 aerobic resistance training circuit improves aerobic fitness and muscle strength in sedentary

3 young females. J. Strength Cond. Res. 29: 1592-1600.

4 Noble, B.J., Borg, G.A.V., Jacobs, I., Ceci, R., and Kaiser, P. 1983. A catergory-ratio

5 perceived exertion scale: relationship to blood and muscle lactates and heart rate. Med. Sci.

$6 \quad$ Sports Exerc. 15: 523-528.

7 Nybo, L., Sundstrup, E., Jakobsen, M.D., Mohr, M., Hornstrup, T., Simonsen, L., Bulow,

8 J., Randers, M.B., Nielsen, J.J., and Aagaard, P. 2010. High-intensity training versus traditional

9 exercise interventions for promoting health. Med. Sci. Sports Exerc. 42: 1951-1958.

10 Ronnestad, B.R., Hansen, J., Vegge, G., Tonnessen, E., and Slettalokken, G. 2015. Short

11 intervals induce superior training adaptations compared with long intervals in cyclists - An

12 effort-matched approach. Scand. J. Med. Sci. Sports. 25: 143-151.

14 accumulation relative to the anaerobic and respiratory compensation thresholds. J. Appl. Physiol:

15 Respirat. Environ. Exercise. Physiol. 54: 13-17.

17 on $\mathrm{VO}_{2} \max$ and aerobic exercise performance: A systematic review and meta-analysis. Scand. J.

18 Med. Sci. Sports. 23: e341-352. 
Tabata I., Nishimura K., Kouzaki M., Hirai Y., Ogita F., Miyachi M., and Yamamoto K.

2 1996. Effects of moderate-intensity endurance and high-intensity intermittent training on

3 anaerobic capacity and $\mathrm{VO}_{2} \mathrm{max}$. Med. Sci. Sports Exerc. 28: 1327-1330.

4

Verdijk, L.B., van Loon, L., Meijer, K., and Savelberg, H.H.C.M. 2009. One-repetition

5 maximum strength test represents a valid measure to assess leg strength in vivo in humans. J.

6 Sports Sci. 27: 59-68.

Weston, M., Taylor, K.L., Batterham, A.M., and Hopkins, W.G. 2014. Effects of low-

8 volume high-intensity interval training (HIT) on fitness in adults: A meta-analysis of controlled

9 and non-controlled trials. Sports Med. 44: 1005-1017.

10 Wong P.L., Chaouachi A., Chamari K., Dellal A., and Wisloff U. 2010. Effect of

11 preseason concurrent muscular strength and high-intensity interval training in professional soccer

12 players. J. Strength Cond. Res. 24: 653-660.

14 2011. Aerobic and anaerobic changes with high-intensity interval training in active college-aged

15 men. The Journal of Strength and Conditioning Research. 25: 1104-1112. 
1 Tables

2 Table 1. Participant characteristics

\section{Row-HIIT MM-HIIT}

Age (years) $\quad 24.3 \pm 5.2 \quad 25.1 \pm 5.6$

Gender Female $(n=14) \quad$ Female $(n=14)$

Height (cm) $\quad 171.6 \pm 7.6 \quad 166 \pm 2.4$

Weight (kg)

$\begin{array}{lll}\text { Pre } & 73.2 \pm 11.9 & 66.6 \pm 9.8 \\ \text { Post } & 73.6 \pm 11.9 & 67.6 \pm 10.2\end{array}$

3

4

5

6

7

8

9

10

11

12

13

14 
1 Table 2. $\mathrm{VO}_{2} \max$ and Wingate anaerobic test data

\begin{tabular}{|c|c|c|c|c|}
\hline & \multicolumn{2}{|c|}{ Row-HIIT } & \multicolumn{2}{|c|}{ MM-HIIT } \\
\hline & Pre & Post & Pre & Post \\
\hline $\begin{array}{l}\mathrm{VO}_{2} \max \\
\left(\mathrm{ml} \cdot \mathrm{kg}^{-1} \cdot \mathrm{min}^{-1}\right)\end{array}$ & $36.7 \pm 4.7$ & $38.3 \pm 4.6^{*}$ & $36.2 \pm 5.7$ & $38.5 \pm 5.4^{*}$ \\
\hline $\begin{array}{l}\mathrm{VO}_{2} \text { at } \mathrm{RCT} \\
\left(\mathrm{ml} \cdot \mathrm{kg}^{-1} \cdot \mathrm{min}^{-1}\right)\end{array}$ & $33.9 \pm 4.7$ & $35.5 \pm 4.7$ & $31.1 \pm 5.7$ & $33.2 \pm 5.6^{*}$ \\
\hline $\begin{array}{l}\mathrm{VO}_{2} \text { at AT } \\
\left(\mathrm{ml} \cdot \mathrm{kg}^{-1} \cdot \mathrm{min}^{-1}\right)\end{array}$ & $28.7 \pm 5.0$ & $31.7 \pm 3.8^{*}$ & $27.2 \pm 6.7$ & $29.7 \pm 5.7 *$ \\
\hline $\begin{array}{l}\text { Anaerobic power } \\
\text { (Watts) }\end{array}$ & $481 \pm 135$ & $536 \pm 133^{*}$ & $424 \pm 93$ & $484 \pm 94^{*}$ \\
\hline $\begin{array}{l}\text { Anaerobic capacity } \\
\text { (Watts) }\end{array}$ & $371 \pm 104$ & $417 \pm 100 *$ & $333 \pm 62$ & $389 \pm 69 *$ \\
\hline
\end{tabular}

4

5

6

7

8

9

10

11 


\section{Figure Legends}

2 Figure 1. Muscle strength test data for (a) 1 RM squat, (b) 1 RM press, and (c) 1 RM deadlift for

3 the MM-HIIT and Row-HIIT groups. *significantly different than corresponding pre-test value, $4 \mathrm{p}<0.05 ; \uparrow$ significantly different than post-test for Row-HIIT, $\mathrm{p}<0.05$.

5

6 Figure 2. Muscle power (a; broad jump) and muscle endurance (b; squat endurance at $70 \% 1$

7 RM pre-training squat) for the MM-HIIT and Row-HIIT groups. * significantly different than

8 corresponding pre-test value, $\mathrm{p}<0.05$; † significantly different than post-test for Row-HIIT, $\mathrm{p}<$ 90.05.

10 
(A)

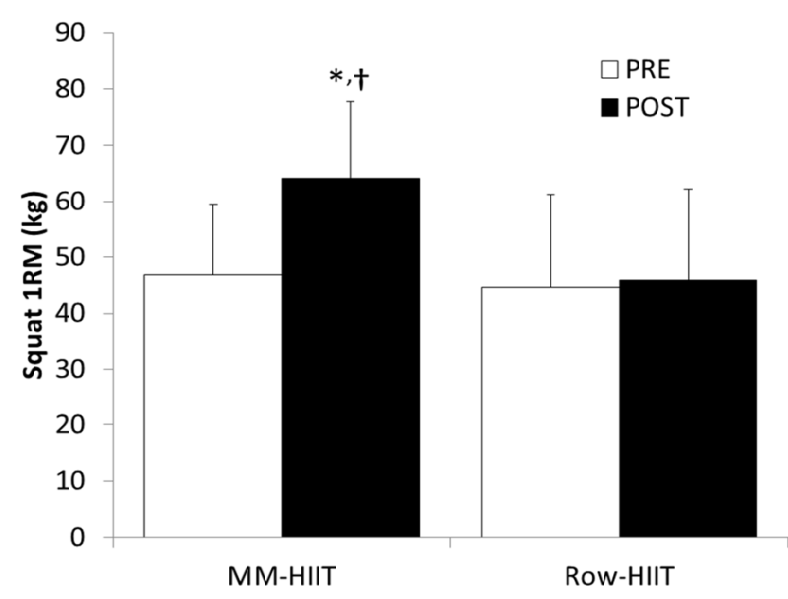

(C)

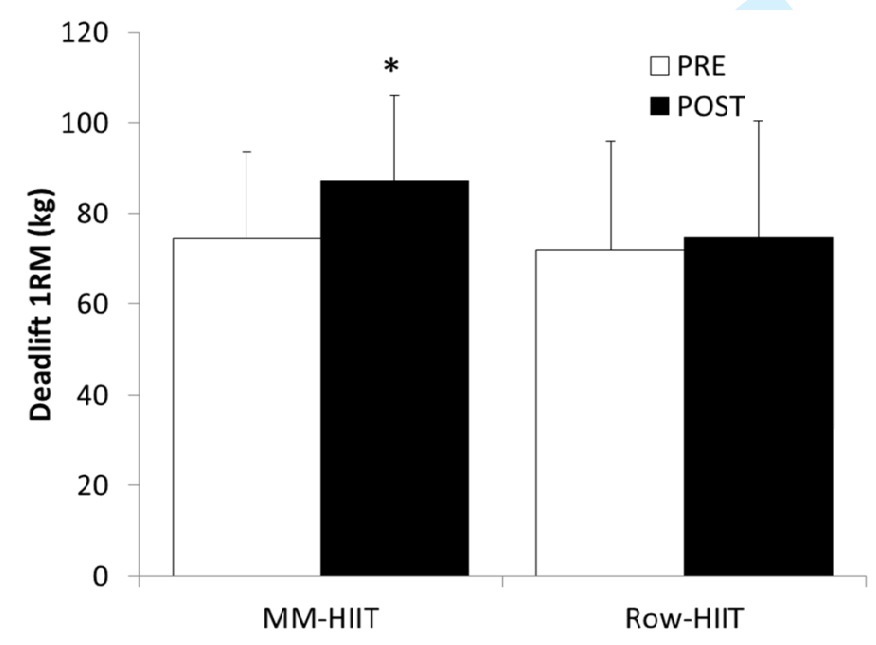

(B)




(A)

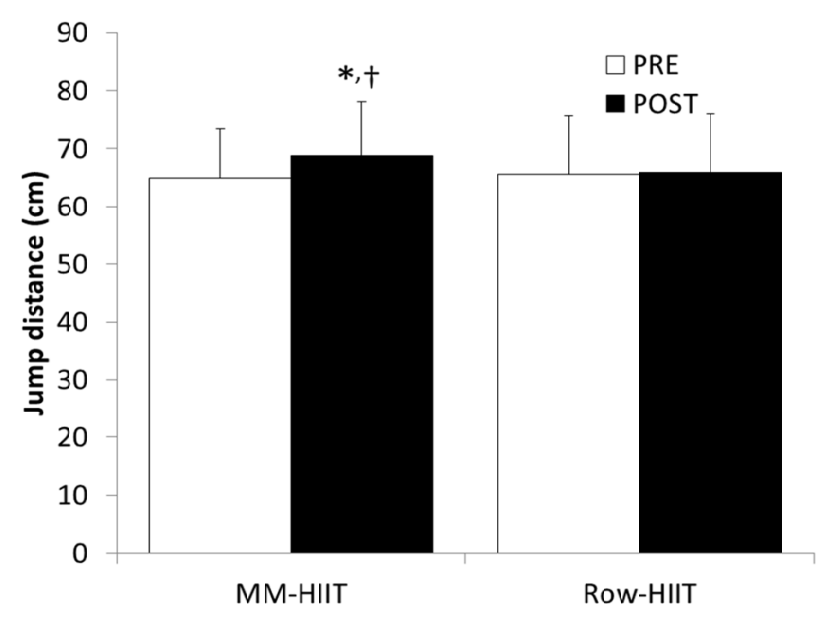

(B)

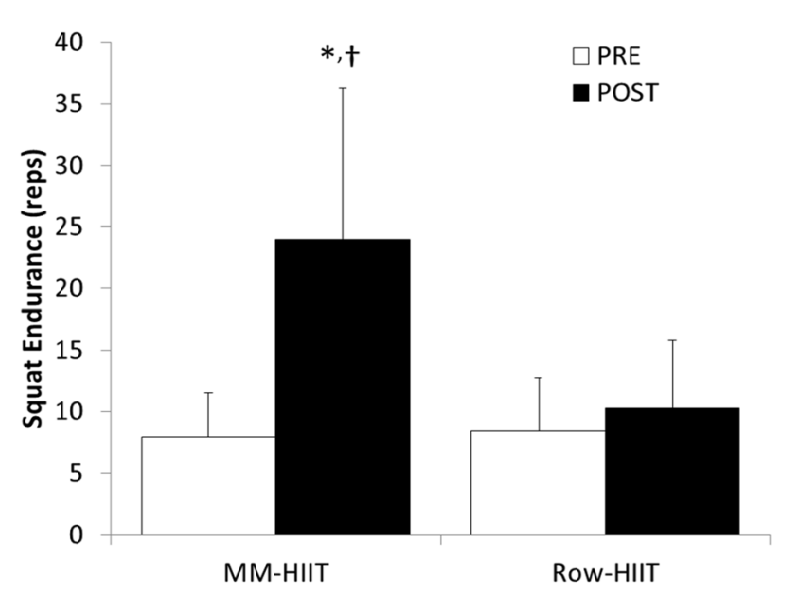

